\title{
Diagnostic Aspiration
}

National Cancer Institute

\section{Source}

National Cancer Institute. Diagnostic Aspiration. NCI Thesaurus. Code C64989.

The withdrawal of fluid via a needle for microscopic examination for the purpose of diagnosing a disease/disorder. 